\title{
A comparison of cyst age and assay method of the efficacy of contact lens disinfectants against Acanthamoeba
}

\author{
Simon Kilvington, Claude Anger
}

\begin{abstract}
Aims-(i) To determine effect of Acanthamoeba cyst age, method of production, and (ii) to assay technique on the efficacy of multipurpose solutions (MPS) and hydrogen peroxide based contact lens disinfectants. (iii) To establish if MPS can remove mature cysts from contact lenses according to the ISO/DIS 14729 regimen test for microbe removal.

Methods-Immature and mature cysts of A polyphaga were tested against the MPS Opti-Free express and the hydrogen peroxide based solutions Oxysept 1Step and Oxysept 1 using two assay methods. Simulated patient regimen testing was performed with the Opti-Free express and Complete using mature cysts inoculated on to group I or group IV lenses.

Results-Immature cysts were sensitive to disinfection by all solutions. No killing was observed with mature cysts with OptiFree express, while immature cysts yielded a 1-2 log reduction in viability. Oxysept 1Step gave a 1.1 (SD 0.3) log reduction in mature cysts after 6 hours. Oxysept 1 gave a $2.4(0.3) \log$ reduction in mature cysts after 4 hours and a $3.8(0.5)$ $\log$ reduction after 6 hours. Patient regimen testing using Opti-Free express and Complete resulted in no recovery of viable mature cysts from the contact lenses or from the soaking solutions.

Conclusion-Cyst age but not method of production used in this study influences the efficacy of contact lens disinfectants against Acanthamoeba. MPS are effective in removing cysts from contact lens surfaces and may have a role in the prevention of acanthamoeba keratitis. (Br F Ophthalmol 2001;85:336-340)
\end{abstract}

Pharmaceutical

Microbiology,

Allergan, Inc, 2525

DuPont Drive, Irvine, CA 92623-9534, USA

C Anger

Correspondence to: Dr Simon Kilvington sk46@leicester.ac.uk

Accepted for publication 4 October 2000

Acanthamoeba is a common soil and water amoeba characterised by a feeding and replicating trophozoite and dormant cyst stage. ${ }^{1}$ The organism is pathogenic to humans causing a severe and potentially blinding keratitis. ${ }^{1-3}$ Contact lens wearers are most at risk from acanthamoeba keratitis and account for approximately $95 \%$ of reported cases. ${ }^{2}{ }^{3}$ Failure to comply with recommended lens cleaning and disinfection instructions and the rinsing or storing of lenses in non-sterile saline solutions or tap water are recognised risk factors. ${ }^{145}$

The resistance of the Acanthamoeba cysts to extremes of temperature, desiccation, and disinfection accounts for the almost ubiquitous presence of the organism in the environment. Surveys have shown that contact lens storage cases may be grossly contaminated with bacteria and fungi in normal use. ${ }^{146}$ This provides a food source for the growth of Acanthamoeba and the organism has been isolated from lens storage cases of symptomatic and asymptomatic lens wearers. ${ }^{14}$

Safe contact lens use requires regular cleaning and disinfection of the lenses and storage in a sterile solution. Ineffective disinfectant solutions or inadequate disinfection times can enable patient contaminant organism survival which, if the lens cases are not cleaned and air dried properly, could subsequently proliferate during contact lens storage. ${ }^{4}{ }^{6}$ To simplify contact lens hygiene and promote better patient compliance, multipurpose solutions (MPS) have been developed. These represent a single solution for the cleaning, disinfection, and storage of contact lenses and hence offer continued antimicrobial protection during lens storage. ${ }^{7}$

The purpose of this study was threefold: (i) to observe the antimicrobial resistance of Acanthamoeba cysts produced from varying growth conditions and maturity to contact lens disinfecting solutions; (ii) to compare the precision and accuracy of two different methods for measuring quantitative Acanthamoeba viability; and (iii) to determine if the recommended International Standards Organisation (ISO) regimen test for removal of microbes from contact lenses would be effective against Acanthamoeba. ${ }^{8}$
Materials and methods

ACANTHAMOEBA STRAINS

Acanthamoeba polyphaga strain ATCC 30873 was used in all studies. The strain was originally isolated from a case of acanthamoeba keratitis in the United Kingdom in 1974. 
Table 1 Contact lens solutions studied

\begin{tabular}{lll}
\hline Test product & Active ingredient & $\begin{array}{l}\text { Recommended disinfection } \\
\text { time }\end{array}$ \\
\hline Opti-Free express (Alcon) & $\begin{array}{l}\text { polyquad 0.001\% and myristamidopropyl } \\
\text { dimethylamine } 0.0005 \%\end{array}$ & 6 hours \\
polyhexanide $0.0001 \%$ & 4 hours \\
Oxysept 1Step (Allergan) & $3 \%$ hydrogen peroxide (one step) & 6 hours \\
Oxysept 1 (Allergan) & $3 \%$ hydrogen peroxide (two step) & 20 minutes-overnight \\
\hline
\end{tabular}

CULTURE

Trophozoites were maintained in an axenic growth medium comprising Biosate (BBL), 20.0 g; glucose, 5.0 g; $\mathrm{KH}_{2} \mathrm{PO}_{4}, 0.3$ g; vitamin $\mathrm{B}_{12}, 10 \mu \mathrm{g}$; L-methionine, $15 \mathrm{mg}$; deionised water to 1 litre. The $\mathrm{pH}$ was adjusted to 6.5-6.6 with $1 \mathrm{~N} \mathrm{NaOH}$ before autoclaving at $121^{\circ} \mathrm{C}$ for 12 minutes. Cysts were produced from axenic trophozoites using Neff's defined, constant $\mathrm{pH}$ encystment medium. ${ }^{9}$ Trophozoites were washed three times in the Neff encystment medium and inoculated into 175 $\mathrm{cm}^{2}$ tissue culture flasks with a filter cap (Nunc, Life Technologies Ltd, Paisley, Scotland) to a final concentration of $\sim 1 \times 10^{5} / \mathrm{ml}$ in $100 \mathrm{ml}$ of medium. The flasks were incubated in air at $32^{\circ} \mathrm{C}$ for $0.5,1$, or 7 days. Cysts were recovered from the flasks by gentle rubbing with a cell scraper and harvested by centrifugation at $1000 \times g$ for 15 minutes. The cysts were then washed three times with $1 / 4$ strength Ringer's solution by centrifugation.

In addition, trophozoites were grown and allowed to encyst on non-nutrient agar plates (1.6\% agar in $1 / 4$ strength Ringer's solution) seeded with the bacterium Escherichia coli (NNA-E coli), strain JM 101. After 7 days of incubation in air at $32^{\circ} \mathrm{C}$, the cysts were recovered by flooding the plates with $1 / 4$ strength Ringer's solution and rubbing firmly with cotton tipped swab. The cysts were concentrated by centrifugation at $500 \times g$ for 10 minutes at room temperature and washed three times with $1 / 4$ strength Ringer's solution by centrifugation to remove the bacteria.

Microscopic examination of the 7 day Neff and NNA-E coli cysts showed $>90 \%$ mature cysts. $^{9}{ }^{10}$ Cysts recovered after 0.5 and 1 day appeared to be $>90 \%$ in the immature developing stage. ${ }^{9} 10$ All cyst preparations were stored at $4^{\circ} \mathrm{C}$ for testing within 7 days.

\section{CONTACT LENS SOLUTIONS}

The contact lens disinfectants studied and the manufacturers' recommended exposure times for disinfection are shown in Table 1. OptiFree express (Alcon) and Complete (Allergan) are multipurpose disinfectants in which a single solution is used for cleaning, disinfecting, rinsing, and storing the lenses. Oxysept 1 Step (Allergan) is a one step 3\% hydrogen peroxide based system where a neutralising tablet is added at the start of disinfection. This slowly inactivates the hydrogen peroxide over a 6 hour period enabling the lenses to be worn without further treatment. Oxysept 1 (Allergan) is a two step hydrogen peroxide based system. Lenses are disinfected for 20 minutes to overnight before the peroxide solution is replaced with a second neutralising solution containing $520 \mathrm{U} / \mathrm{ml}$ catalase.
PATIENT REGIMEN TESTING

Cysts were obtained by 7 day incubation of trophozoites in Neff's encystment medium. The contact lenses tested were new and unworn, Aspect Frequency Disposables (group I: $61.4 \%$ poly, [2-hydroxyethyl methacrylate] $38.6 \%$ water) and Johnson and Johnson Surevue lenses (group IV: 42\% etafilcon A, $58 \%$ water). The lenses reflect high and low water content types. The disinfectant solutions were Opti-Free express and Complete.

Briefly, $10 \mu \mathrm{l}$ aliquots of $1 \times 10^{5}$ cysts in $1 / 4$ strength Ringer's solution were allowed to adhere to both sides of a lens for 5-10 minutes and then subjected to rubbing (20 seconds) and rinsing (10 seconds each lens side) with the MPS and disinfectant soaking (4 hours for Complete and 6 hours for Opti-Free express) in the storage cases supplied by the solution manufacturer. Each lens group was tested in quadruplet. Following soaking, each lens was removed from the storage case and placed into the well of a six place microtitre plate containing $3 \mathrm{ml}$ of ATCC 711 agar (American Type Culture Collection, Rockville, MD, USA). The lens was then overlaid with $1 \mathrm{ml}$ of ATCC 712 soft agar seeded with $E$ coli $\left(1 \times 10^{6} / \mathrm{ml}\right)$ and allowed to set. The plates were then incubated in sealed polythene bags at $32^{\circ} \mathrm{C}$ for 7 days and observed daily for Acanthamoeba plaques in the bacterial lawn, which were then counted.

Using a sterile pipette, $1.0 \mathrm{ml}$ of test lens soaking solution from the storage case was removed from the storage case and $0.2 \mathrm{ml}$ added to each of five wells of a six well microtitre plate containing ATCC 711 agar. The fluid was allowed to absorb to dryness and ATCC 712 soft agar seeded with $E$ coli cells (1 $\times 10^{6} / \mathrm{ml}$ ) overlaid. The plates were then incubated in sealed polythene bags at $32^{\circ} \mathrm{C}$ for 7 days and the number of Acanthamoeba plaques counted.

DISINFECTANT EFFICACY TESTING

Cysts were derived from trophozoite cultures on NNA-E coli culture for 7 days and from incubation in Neff's encystment medium after $0.5,1$, and 7 days. The test disinfectant solutions were Opti-Free express, Oxysept 1Step, and Oxysept 1, with 1/4 strength Ringer's solution used as a control. MPS assays were performed in unused $50 \mathrm{ml}$ polypropylene tubes (Becton Dickinson, Oxford, UK) and the manufacturer's lens storage cases for Oxysept 1Step and Oxysept 1. The polypropylene tubes were "aged" overnight by filling with $15 \mathrm{ml}$ of test solution, which was then discarded, before the tubes were used for testing. With Oxysept 1Step the manufacturer's neutralising tablet (catalase $0.1 \mathrm{mg} /$ tablet) was added to the storage case immediately after cyst inoculation. The disinfectant neutralisers were $0.05 \%$ Tween 80 in $1 / 4$ strength Ringer's solution for Opti-Free express and $0.02 \%$ bovine liver catalase (Sigma Chemical Company, Dorset, England) in $1 / 4$ strength Ringer's solution for Oxysept 1Step and Oxysept 1.

Two methods for assessing disinfectant efficacy were compared: (i) a 96 well microtitre plate assay and (ii) an agar overlay plaque 
Table 2 Comparison of cyst age and assay method on disinfection efficacy (log reduction) after 6 hours of exposure

\begin{tabular}{|c|c|c|c|c|c|c|}
\hline \multirow[b]{3}{*}{ Cyst type and age } & \multicolumn{6}{|c|}{ Disinfectant, assay method, and log cyst kill } \\
\hline & \multicolumn{2}{|c|}{ Opti-Free express } & \multicolumn{2}{|c|}{ Oxysept 1Step } & \multicolumn{2}{|c|}{ Oxysept 1} \\
\hline & $I^{\star}$ & $\mathrm{II} \dagger$ & I & II & I & II \\
\hline Neff 0.5 day & 2.0 & 2.0 & 1.8 & 1.8 & 3.8 & 4.8 \\
\hline Neff 1 day & 1.5 & 1.1 & 2.0 & 1.9 & 3.5 & 4.8 \\
\hline Neff 7 day & 0.3 & 0.3 & 1.5 & 0.8 & 3.3 & 3.5 \\
\hline NNA-E coli 7 day & 0.5 & 0.3 & 1.3 & 0.8 & 4.3 & 3.7 \\
\hline
\end{tabular}

${ }^{\star}$ Microtitre plate assay; †agar overlay plaque assay.

assay. ${ }^{11}$ In each case, cysts were adjusted to a concentration of approximately $1 \times 10^{7} / \mathrm{ml}$ in $1 / 4$ strength Ringer's solution and $100 \mu$ added to $10 \mathrm{ml}$ of disinfectant or control.

For the microtitre plate method, at time intervals of $0,1,2,4,6,8$, and 24 hours, $100 \mu \mathrm{l}$ was removed and added to $900 \mu \mathrm{l}$ of appropriate neutraliser. Serial 10 -fold dilutions in $1 / 4$ strength Ringer's solution $(20 \mu \mathrm{l}+180 \mu \mathrm{l})$ were made in triplicate across the wells of a microtitre plate. Twenty five $\mu \mathrm{l}$ of $E$ coli $\left(\mathrm{OD}_{600} 0.4\right)$ were added to each well and the plate sealed and incubated in air at $32^{\circ} \mathrm{C}$. Plates were inspected daily for 7 days for the presence of amoebal growth (encystment and trophozoite replication) in the wells. The number of surviving organisms at each time interval was determined by Reed and Muench computation

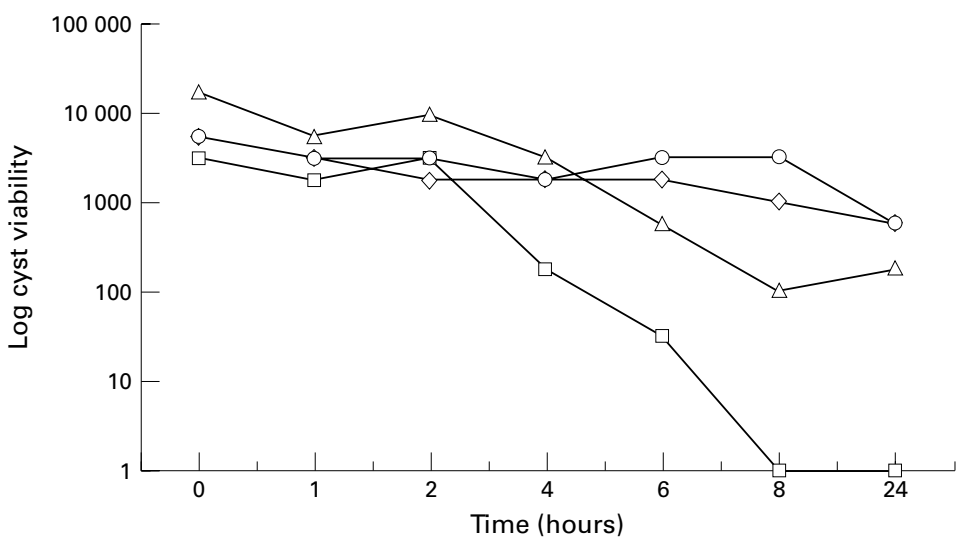

Figure 1 Efficacy of multipurpose solution Opti-Free express against Acanthamoeba polyphaga (ATCC 308730) cysts. Square = Neff 0.5 day; triangle = Neff 1 day; circle = Neff 7 day; diamond $=$ NNA-E coli.

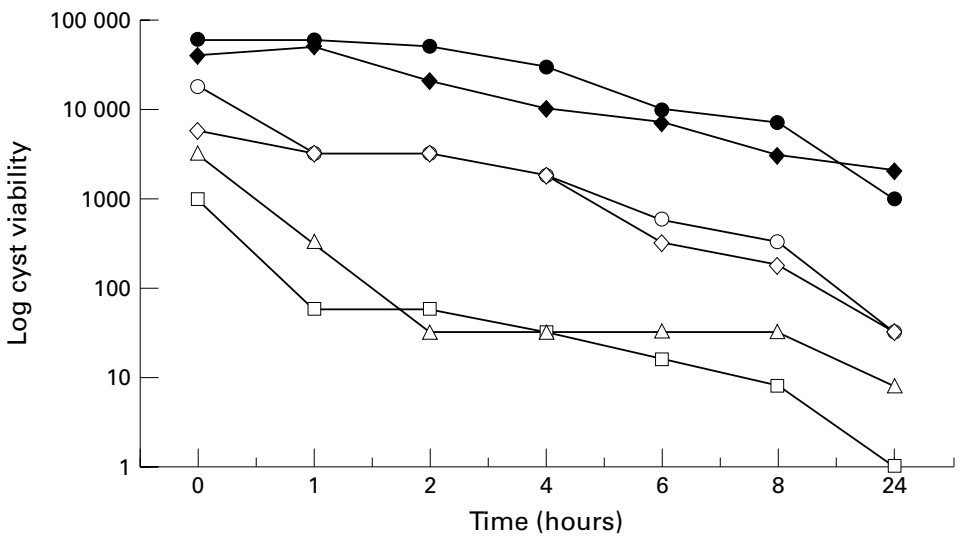

Figure 2 Efficacy of hydrogen peroxide system Oxysept 1Step against Acanthamoeba polyphaga (ATCC 308730) cysts. Microtitre plate assay. Open square = Neff 0.5 day; open triangle $=$ Neff 1 day; open circle $=N e f f 7$ day; open diamond $=N N A-E$ coli. Agar overlay plaque assay: solid circle Neff 7 day; solid diamond NNA-E coli). as described previously for Acanthamoeba cyst viability enumeration. ${ }^{12} 13$

In the agar overlay plaque assay, at $0,1,2,4$, 6,8 , and 24 hours, $1 \mathrm{ml}$ was removed and added to $9 \mathrm{ml}$ of appropriate neutraliser. Serial 10 -fold dilutions in $1 / 4$ strength Ringer's solution were made and $1 \mathrm{ml}$ of each dilution spread as $0.2 \mathrm{ml}$ aliquots over five Petri dishes containing ATCC 711 agar. After allowing to absorb for 20 minutes, the plates were overlaid with ATCC 712 soft agar containing $E$ coli cells $\left(1 \times 10^{6} / \mathrm{ml}\right)$ and allowed to set. The plates were incubated in air at $32^{\circ} \mathrm{C}$ for 7 days and the number of Acanthamoeba plaques counted.

\section{Results}

PATIENT REGIMEN TESTING

Following the patient simulated in-use test procedure with Opti-Free express and Complete no viable Acanthamoeba could be cultured from the group 1 (low water content) or group IV lenses (high water content). Neither could viable Acanthamoeba be cultured from the soaking solutions after the manufacturer's recommended times of 6 and 4 hours respectively (results not shown).

\section{DISINFECTANT EFFICACY}

Of the two methods tested for estimating Acanthamoeba cyst viability, the microtitre plate assay and agar overlay plaque assay gave comparable results regardless of cyst preparation method or age (Table 2: p >0.05 by ANOVA). One difference was noted with Oxysept 1 Step against NNA-E coli or 7 day Neff cysts. Here, a 0.8 log reduction was recorded after 6 hours by the agar overlay plaque assay compared with 1.3-1.5 log units in the microtitre plate method. However, this difference was not statistically significant ( $p$ $>0.05$ ).

The results for both the microtitre plate and plaque agar assays on the activity of Opti-Free express and Oxysept 1Step (one step) and Oxysept 1 (two step) against the various cyst preparations are shown in Figures 1-3. Overall, this showed that immature cysts, derived from 0.5 or 1 day incubation in Neff's encystment medium, were markedly more sensitive to disinfection than the mature forms obtained after 7 days of incubation in this medium or from culture on NNA-E coli agar ( $\mathrm{p}<0.05)$. No difference in sensitivity or resistance was found between the NNA-E coli and the 7 day Neff's cysts ( $p>0.05)$.

After 6 hours of exposure (the manufacturers' recommended disinfection time for both Opti-Free express and Oxysept 1Step) the reduction in cyst viability by Opti-Free express against $0.5,1$, and 7 day Neff medium produced cysts was 2, 1.5, and $0.3 \log$ units respectively. For Oxysept 1 Step the respective reduction in cyst viability was $1.8,2$, and 1.5 $\log$ units. For NNA-E coli prepared cysts the values were $0.5 \mathrm{log}$ units with Opti-Free express and $1.3 \mathrm{log}$ units with Oxysept 1Step. The peroxide Oxysept 1 system was the most effective disinfectant against the mature 7 day Neff and NNA-E coli cysts, giving a 1.8-2.0 log reduction after 2 hours of exposure and 


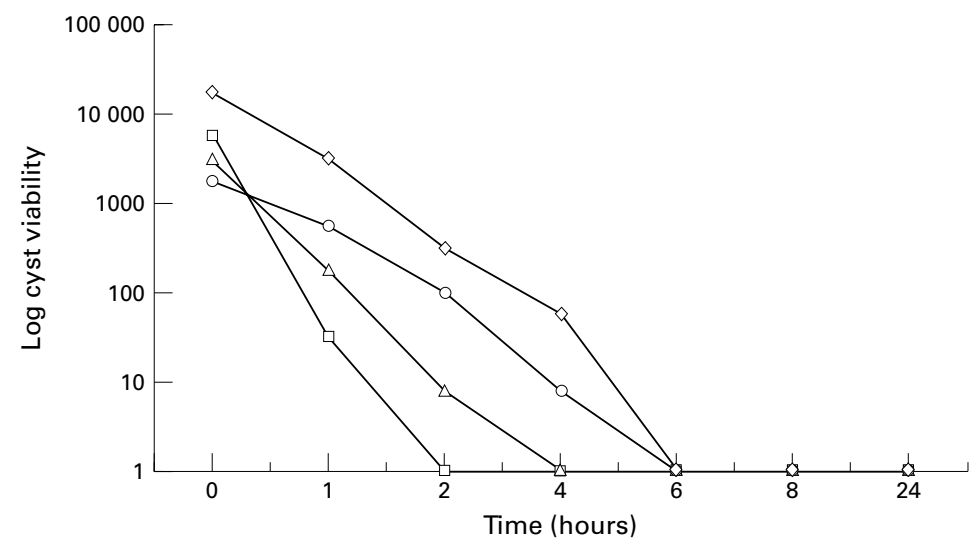

Figure 3 Efficacy of hydrogen peroxide system Oxysept 1 against Acanthamoeba polyphaga (ATCC 308730) cysts. Square = Neff 0.5 day; triangle = Neff 1 day; circle = Neff 7 day; diamond $=N N A-E$ coli.

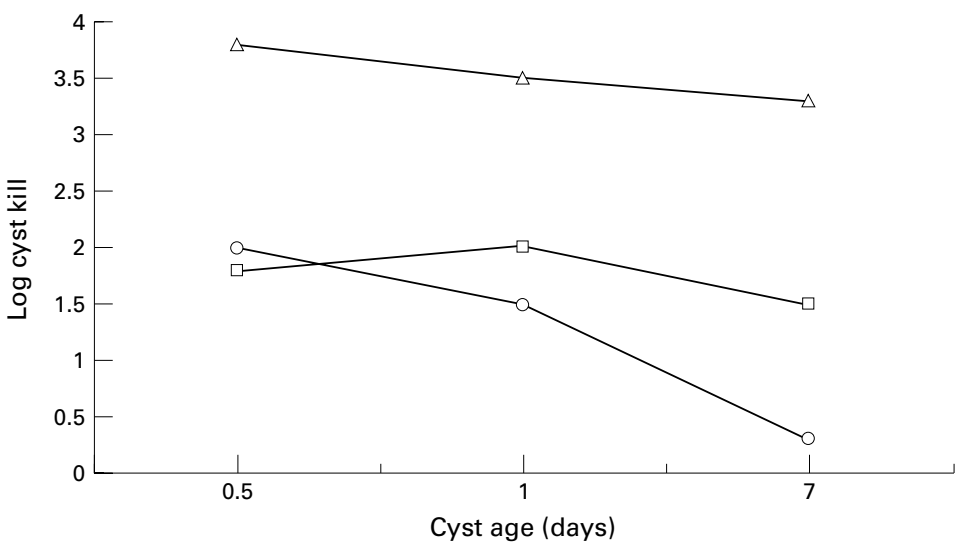

Figure 4 Effect of cyst age (Neff's medium prepared) on efficacy of Oxysept 1 (triangle), Oxysept 1Step (square), and Opti-Free express(circle) disinfectants after 6 hours of exposure.

$2.4-2.5$ by 4 hours (Fig 3). At 6 hours, total kill of the challenge inoculum had occurred resulting in a 3.3-4.3 log reduction.

A summary of the disinfectant activity after 6 hours of exposure against the various cyst forms is given in Table 2 and Figure 4. This shows the marked effect of cyst age on disinfection by Opti-Free express and, to a lesser degree, Oxysept 1Step. This relation was not seen with Oxysept 1 as total kill of all the cyst forms had occurred by 6 hours (Fig 3). Overall, mature cysts produced by 7 days' incubation in either Neff's encystment medium or the NNA-E coli plate culture yielded identical resistance to all of the contact lens disinfection solutions tested in this study.

All control experiments in which the respective disinfectant solutions were replaced with $1 / 4$ strength Ringer's solution resulted in $<0.3 \mathrm{log}$ reduction in viability for all cyst types after 24 hours of exposure (results not shown).

\section{Discussion}

Disinfection is a fundamental part of the contact lens hygiene regime and serves to prevent the growth of potentially pathogenic organisms that may lead to ocular infections. ${ }^{14}{ }^{15}$ Non-compliant or incomplete disinfection may allow survival of bacteria and fungi that can provide a food source inside the contact lens storage case for the growth and replication of Acanthamoeba. ${ }^{4-6}$ From here, the organism can adhere to the contact lens for inoculation on to the cornea to cause infection.

A key objective of this study was to investigate the effect of cyst age and method of production on the efficacy of contact lens disinfection. During encystment, Acanthamoeba organisms pass through stages of preencystment trophozoites (rounded cells), immature cysts (poorly developed walls), and mature cysts (clearly defined inner and outer walls). Using a defined encystment medium, ${ }^{9}$ Acanthamoeba from these stages of cyst development were tested for their susceptibility to disinfection. The findings were compared with mature cysts obtained from trophozoites grown on NNA-E coli medium. The results showed that immature cysts derived from 0.5 or 1 day incubation in the Neff encystment medium were significantly more sensitive to disinfection that mature forms obtained after 7 days or from NNA-E coli culture.

For example, Opti-Free express gave a reduction in cyst viability of 2 and $1.1-1.5 \mathrm{log}$ units for 0.5 and 1 day old cysts after 6 hours of exposure but gave $\leqslant 0.5 \mathrm{log}$ unit reduction for the same time with mature cysts.

Opti-Free express has been reported to be effective against Acanthamoeba cysts, giving a $2-3 \log$ reduction in viability after 6 hours. ${ }^{16}{ }^{17}$ The findings are in contrast with the results of this study in which no significant cysticidal activity was observed. In the previous studies with Opti-Free express, trophozoites were left to encyst during prolonged incubation in a nutrient rich broth (proteose peptone-yeast extract-glucose medium: PYG) compared with the chemically defined medium used here. ${ }^{16} 17$ Possibly, morphologically mature cysts produced by extended incubation of trophozoites in nutrient rich PYG medium either lack or possess additional cell wall materials making them more susceptible to chemical disinfection. Previous work has shown that immature cysts produced in PYG $+50 \mathrm{mM} \mathrm{MgCl}$ are more sensitive to the biocides polyhexamethylene biguanide (PHMB) and chlorhexidine than mature forms. ${ }^{10}$ This observation is in accord with the findings of this study in which immature cysts were markedly more susceptible to disinfection that the mature forms. Electron microscopic studies of PYG cyst preparations indicates that morphologically mature forms are produced, however their physiological variances from other mature cyst forms is not known. ${ }^{18}$

Subsequent to this study, it was reported that the ingredient in Opti-Free express of $0.0005 \%$ myristamidopropyl dimethylamine (MAPD) adheres to certain plastics. ${ }^{18}$ In this study, testing was conducted in polypropylene tubes previously aged in Opti-Free express compared with virgin glass used previously. ${ }^{16}{ }^{17}$ Further studies are indicated to investigate the effect of test materials and method of cyst production on the disinfectant activity of MAPD against Acanthamoeba.

A similar effect of cyst age on disinfectant susceptibility was also noted here with the hydrogen peroxide systems. For the Oxysept 
1Step system, 6 hour exposure gave reductions in viability for $0.5,1$, and 7 day Neff medium cysts of $1.8,2.0$, and $1.5 \log$ units, respectively. For the Oxysept 1 solution, 2 hours of exposure gave reductions in viability for $0.5,1$, and 7 day Neff medium cysts of 3.8, 2.6, and $2.0 \mathrm{log}$ units, respectively. As was found with Opti-Free express, immature cysts are therefore more susceptible to hydrogen peroxide disinfection than mature ones. By 4 hours, total kill of the cyst challenge inoculum had occurred. This study confirms, therefore, previous findings on the efficacy of 3\% hydrogen peroxide against Acanthamoeba cysts providing adequate exposure times are used prior to neutralisation. ${ }^{15} 19$ The manufacturer's recommended disinfectant time for Oxysept 1 before neutralisation is 20 minutes to overnight before the solution is replaced with a second catalase neutralising solution. From the findings of this study, the minimum recommended disinfection time with this solution should be extended to at least 2-4 hours before neutralisation to ensure killing of Acanthamoeba cysts.

Previous studies have demonstrated that Acanthamoeba trophozoites and cysts can adhere to the surface of contact lenses and that the exposure time and suspending medium affects the rate of attachment. ${ }^{20-22}$ In this study, it was shown that $5 \log$ units of Acanthamoeba cysts inoculated on to the surface of group I or group IV contact lenses could be completely removed by cleaning, rinsing, and soaking in the multipurpose solutions Opti-Free express and Complete. Previous studies have shown that Acanthamoeba adhere better to high water content lenses (group IV) than low water types (group I). ${ }^{2122}$ Although adherence was not investigated here, the experiments were conducted with cysts suspended in $1 / 4$ strength Ringer's solution and allowed to be in contact with the lenses for 10 minutes. This is likely to be an insufficient time to allow Acanthamoeba adherence and the findings probably relate only to amoebae that have settled, rather than truly attached, to the lens surfaces.

Unlike the requirements for efficacy testing of contact lens disinfectants against bacteria and fungi, ${ }^{8}$ no standard protocol exists for Acanthamoeba cysts and a variety of protocols have been used. ${ }^{1115171923}$ Besides the influence of cyst age or production method, the assay technique could also influence the results. The results of this study show that both the microtitre plate assay and the agar overlay plaque assay give comparable results. The microtitre plate assay is simpler to perform but gives only statistical probabilities of cyst viable numbers at a given dilution. ${ }^{12}{ }^{13}$ This compares with the agar overlay plaque assay which enumerates actual cyst viable numbers for a given dilution but is more demanding to perform. ${ }^{11}$ Further studies are clearly indicated to address further the factors of cyst age, preparation method, assay materials and methods with a view to establishing reliable and reproducible standard assays for Acanthamoeba disinfectant testing. ${ }^{8}$

Acanthamoeba keratitis is a rare infection and recent surveys indicate that the incidence of infection is declining in England. ${ }^{2}$ However, the almost ubiquitous distribution of Acanthamoeba in the environment presents a constant challenge to the contact lens wearer. ${ }^{13}$ The combination of patient compliance, removal of tap water from lens care regimens, and correct use of MPS and hydrogen peroxide disinfectants should contribute to a continued decline in acanthamoeba keratitis cases.

This work was supported, in part, by a grant in aid of research to S Kilvington from Allergan, Inc, USA.

1 Kilvington S, White DG. Acanthamoeba: biology, ecology and human disease. Rev Med Microbiol 1994;5:12-20.

2 Radford CF, Lehmann OJ, Dart JKG for the National Acanthamoeba Keratitis Study Group. Acanthamoeba keratitis: multicentre survey in England 1992-6. Brf Ophthalmol 1998;82:1387-92.

3 Radford CF, Bacon AS, Dart JKG, et al. Risk factors for Acanthamoeba keratitis in contact lens users: a case control study. BMF 1995;10:1567-70.

4 Gray TB, Cursons TM, Sherwan JF, et al. Acanthamoeba, bacterial, and fungal contamination of contact lens storage cases. Brf Ophthalmol 1995;79:601-5. 5 Larkin DFP, Kilvington S, Easty, DL. Contamination of contact lens storage cases by Aca
Br f Ophthalmol 1990;74:133-5.

6 Bottene E, Madayag RM, Qureshi MN. Acanthamoeba keratitis: synergy between amebic and bacterial cocontaminants in contact lens care systems as a prelude to infection. f Clin Microbiol 1992;30:2447-50.

7 Stevenson RWW, Seal DV. Has the introduction of multi-purpose solutions contributed to a reduction in Acanthamoeba keratitis in contact lens wearers?: a review. Contact Lens Anterior Eye 1998;21:89-92.

8 ISO/DIS 14729. Ophthalmic optics-contact lens care products-microbiological requirements and test methods for products and regimens for hygienic management of contact lenses. 1999.

9 Neff RJ, Ray SA, Benton, WF, et al. Induction of synchronous encystment (differentiation) in Acan-
thamoeba sp. Methods Cell Physiol 1964;1:55-83.

10 Khunkitti W, Lloyd D, Furr JR, et al. Acanthamoeba castellanii: growth, encystment, excystment and biocide castellanii: growth, encystment, excys
susceptibility. F Infection 1998;36:43-8.

11 Anger C, Rupp D, Pomrenke M, et al. A quantitative bacterial plaque assay for the enumeration of viable Acanthamoeba cells. Rev Infect Dis 1991;13 (supp1 5):S396.

12 Reed LJ, Muench H. A simple method for estimating fifty percent endpoints. Am f Hyg 1938;27:493-7.

13 Buck SL, Rosenthal RA. A quantitative method to evaluate neutralizer toxicity against Acanthamoeba castellanii. Appl Environmental Microbiol 1996;62:3521-6.

14 Gyulai P, Dziabo A, Kelly W, et al. Relative neutralization ability of six hydrogen peroxide disinfection systems. Contact Lens Spectrum 1987; May:61-5.

15 Silvany RE, Doughterty JM, McCulley JP, et al. The effect of currently available contact lens disinfection systems on Acanthamoeba castellanii and Acanthamoeba polyphaga. Ophthalmology 1990;7:286-90.

16 Kilvington S. Reducing the risk of microbial keratitis in soft contact lens wearers. Optician 1998;216:28-31.

17 Buck SL, Rosenthal RA, Abshire, RL. Amoebicidal activity of a preserved contact lens multipurpose disinfecting solution compared with a disinfection/neutralisation peroxide system. Contact Lens Anterior Eye 1998;21:81-4.

18 Van Duzee B, Schlech B. The activity of a multi-purpose solution against Acanthamoeba. Optician 1999;217:34-5.

19 Kilvington S. Activity of water biocide chemicals and contact lens disinfectants on pathogenic free-living amoebae. Int Biodeterioration 1990;26:127-38.

20 John T, Desai D, Sahm D. Adherence of Acanthamoeba castellanii cysts and trophozoites to unworn soft contact lenses. Am ₹ Opthalmol 1989;108:658-64.

21 Kilvington S, Larkin F. Adherence of Acanthamoeba to contact lenses and their removal following cleaning. Eye 1990;4:589-93.

22 Kilvington S. Acanthamoeba trophozoite and cyst adherence to four types of soft contact lens and removal by ence to four types of soft contact
cleaning agents. Eye 1993;7:535-8.

23 Hugo ER, McLaughlin WR, Oh KH, et al. Quantitative enumeration of Acanthamoeba for evaluation of cyst inactivation in contact lens solutions. Invest Ophthalmol Vis Science 1991;32:655-65. 